\title{
Oxidative stress, DNA damage and the Y chromosome
}

\author{
R. John Aitken ${ }^{1}$ and Csilla Krausz ${ }^{2 *}$ \\ ${ }^{1}$ School of Biological and Chemical Sciences, Centre for Life Sciences, University of \\ Newcastle, University Drive, Callaghan, NSW 23008, Australia; and ${ }^{2}$ Immunogenetique \\ Humaine, Institut Pasteur, 25 rue du Dr Roux, 75724 Paris, Cedex 15, France
}

\begin{abstract}
Recent advances in understanding of male infertility have implicated two major causative factors, oxidative stress and $Y$ chromosome deletions. A major cause of oxidative stress appears to be the high rate of reactive oxygen species generation associated with the retention of excess residual cytoplasm in the sperm midpiece. Other possible causes include the redox cycling of xenobiotics, and antioxidant depletion or apoptosis. Oxidative stress induces peroxidative damage in the sperm plasma membrane and DNA damage in both the mitochondrial and nuclear genomes. Nuclear DNA damage in the germ line of the father may be associated with pathology in the offspring, including childhood cancer and infertility. Gene deletions on the non-recombining region of the $Y$ chromosome account for the infertility observed in about $15 \%$ of patients with azoospermia and $5-10 \%$ of subjects with severe oligozoospermia. The $Y$ chromosome is particularly susceptible to gene deletions because of the inability of the haploid genome to deploy recombination repair in retrieving lost genetic information. Aberrant recombination, defective chromatin packaging, abortive apoptosis and oxidative stress may all be involved in the aetiology of DNA damage in the germ line. The factors responsible for $Y$ chromosome deletions in spermatozoa remain unresolved but may be one facet of a central reproductive problem: controlling the amount of oxidative stress experienced by germ cells during their differentiation and maturation in the male reproductive tract.
\end{abstract}

The testis is the engine of evolution (Short, 1997; Hales et al., 1999). The genetic variation that underpins the evolutionary process appears to be created predominantly in the male germ line as a consequence of a mutagenic machinery that is driven by a variety of replicationdependent and -independent factors (Agulnik et al., 1997). Reproduction in human males in particular is characterized by the production of large numbers of spermatozoa by a spermatogenic process that has scant regard for the phenotypic normality of the gametes or their genomic integrity. Human sperm chromatin is often poorly compacted (Sakkas et al., 1999a) and frequently contains DNA strand breaks (Irvine et al., 2000). In addition, the likelihood of damage to the mitochondrial genome during the differentiation and functional lifespan of a spermatozoon is so great that these structures are ubiquitinated and destroyed in the oocyte after fertilization, to overcome the risk of them contributing to the embryonic mitochondrial pool (Kao et al., 1998; Reynier et al., 1998; Cummins, 2000). Although uniparental inheritance of mitochondria is common to nearly all eukaryotes (Birky, 1995), populations of human spermatozoa exhibiting evidence of mitochon-

Email: jaitken@mail.newcastle.edu.au

*Present address: Andrology Unit, Unversity of Florence, Vle. Pieraccini, 6, Florence, Italy. drial dysfunction also show high rates of nuclear DNA fragmentation (Donnelly et al., 2000).

In contrast to the mass production and genetic instability that characterize the male gamete (Box 1), the female germ line is a model of constancy. Female gametes do not replicate in the adult and spend most of their life in a state of relative dormancy as primordial follicles. This quiescent state is only broken for a brief period when the oocytes enter the growing follicle pool just before ovulation. As a result of this lack of cell division and intense metabolic activity, the ratio $\left(\alpha_{\mathrm{m}}\right)$ of male to female mutations in primates is thought to be about 3-6 for humans and higher primates. For example, Shimmin et al. (1993) analysed the intron sequences associated with $X$ - and $Y$-linked zinc finger genes $(Z F X / Z F Y)$ and recorded a relatively high $\alpha_{\mathrm{m}}$ value of 6 . This value may have been influenced by the fact that the pattern of $Z F X / Z F Y$ expression is known to vary among species. As a consequence, it is possible that the functional significance of this zinc finger protein has changed with time and distortions in mutation rate have been introduced as a consequence of differential selection pressure. Agulnik et al. (1997) addressed this criticism by selecting the SMCY/ SMCX gene pair. The SMC gene encodes a minor transplantation antigen that is expressed in all tissues studied and is present in mice, men and marsupials. Significantly, this gene escapes $X$ inactivation, indicating that the $X$ and $Y$ 


\section{Box 1. Evolution of the $Y$ chromosome}

The human $\mathrm{Y}$ chromosome derives largely from a single autosomal region that was added to the sex chromosomes 80-130 million years ago (Waters et al., 2001). The original $\mathrm{X}$ and $\mathrm{Y}$ chromosomes were homologous (Delbridge and Graves, 1999). However, most of the genes from the original sex chromosome have decayed on the $Y$ chromosome such that the latter now contains just a small number of genes (largely performing housekeeping functions) with homologues on the $X$ chromosome. The tendency for genes to degenerate on the $Y$ chromosome is not unique to humans; indeed, there has been a general evolutionary tendency for non-paired sex chromosomes to degenerate and even (in some species of Drosophila) to disappear entirely (Lahn and Page, 1997). Many of the remaining functional genes on the human $Y$ chromosome appear to have evolved from autosomal homologues, or in one case $(C R Y)$ retroposition of the corresponding mRNA (Saxena et al., 1996; Chai et al., 1997; Lahn and Page $1997,1999)$. Once located on the $Y$ chromosome, there has been a strong tendency for these genes to undergo amplification, presumably because the presence of multiple gene copies creates a buffer against attrition (Burgoyne, 1998). These Y chromosome-specific genes encode molecules that are essential for sex determination $(S R Y)$ or male fertility (RBM, DAZ) (Delbridge and Graves, 1999; Marshall Graves, 2000).

copies of SMC must be functionally interchangeable, to maintain equal gene dosage in males and females. This functional consistency makes the $S M C Y / S M C X$ gene pair an excellent candidate for calculating $\alpha_{\mathrm{m}}$. Analysis of these genes in mice, humans and horses revealed that the SMCY copy of the gene is evolving 1.8 times more quickly than $S M C X$. An $\alpha_{\mathrm{m}}$ value of approximately 3 for this gene supports the concept of male-driven evolution. This value is sufficiently small for the mutations to be induced by mechanisms other than replication error, indicating a role for additional factors in the induction of DNA mutation in the male germ line, including methylation and free radical generation (Agulnik et al., 1997).

These observations raise questions about the origins and nature of DNA damage in the male germ line and the impact such damage has on fertility, embryonic viability and the subsequent health and fertility of offspring. These proposed associations form the basis of this review.

\section{Nature of DNA damage in the germ line}

Two types of DNA damage appear to characterize the male germ line: replication errors and DNA fragmentation.

\section{Replication errors}

Since spermatogonial stem cells replicate throughout life, the spermatozoa of older men will have a history involving many more cell divisions than the gametes of younger men. As a consequence, the gametes of older men can be expected to exhibit a proportionately higher incidence of mutations as a result of replication errors. It is for this reason that the occurrence of dominant genetic disease (for example, Apert's syndrome, achondroplasia) in a child with genetically normal parents invariably involves a mutation in the germ line of the father and is strongly correlated with paternal age (Crow, 1997).

\section{DNA fragmentation}

This kind of damage is characterized by single and double DNA strand breaks and is particularly frequent in the ejaculates of subfertile men (Irvine et al., 2000). Of course, DNA fragmentation does not constitute a mutation in its own right but it is a promutagenic change that has the potential to generate mutations in offspring as a consequence of inadequate or defective repair. Such damage comes from three potential sources: oxidative stress, abortive Fas-mediated apoptosis or deficiencies in natural processes such as recombination and chromatin packaging that involve the induction of DNA strand breaks.

\section{Oxidative stress in the male germ line}

The susceptibility of male germ cells to oxidative stress has been appreciated since MacLeod (1943) observed that human spermatozoa incubated under high oxygen tensions in vitro lost motility via mechanisms that could be reversed by the presence of catalase in the incubation medium. MacLeod (1943) concluded from these experiments that human spermatozoa must be able to generate hydrogen peroxide from ground state oxygen and that the former must be damaging to sperm viability. Jones et al. (1979) demonstrated subsequently that the mechanism by which oxidative stress induced motility loss in mammalian spermatozoa involved the induction of peroxidative damage to the sperm plasma membrane. Human spermatozoa are particularly vulnerable to such stress because their plasma membranes are so enriched with unsaturated fatty acids, particularly decosohexaenoic acid with six double bonds per molecule (Jones et al., 1979). These unsaturated fatty acids are essential to give the plasma membrane the fluidity it needs to participate in the membrane fusion events associated with fertilization. However, when reactive oxygen species attack the double bonds associated with unsaturated fatty acids, a lipid peroxidation chain reaction is initiated that if not arrested leads to a loss of membrane fluidity and a consequent loss of sperm function (Fig. 1).

There are two important features of this peroxidative damage that are relevant to the aetiology of male infertility. Firstly, the lipid peroxidation cascade is catalysed by transition metals such as iron and copper (Jones et al., 1979; Aitken et al., 1993). Addition of ferrous iron to suspensions of human spermatozoa results in a dose-dependent acceleration of lipid peroxidation and a concomitant decrease in the motility and fertilizing potential of these cells (Aitken 
(a) $y=-10.934 x+71.428, r=0.824$

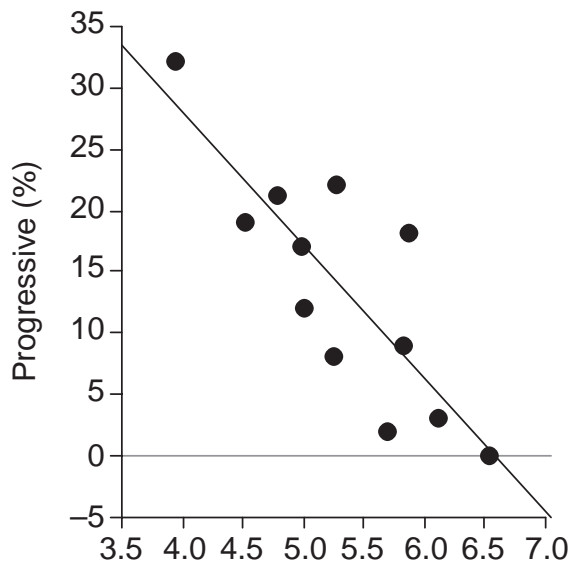

Log PMA-basal chemiluminescence (counts $\mathrm{min}^{-1}$ )

(c) $y=-34.084 x+221.988, r=0.891$

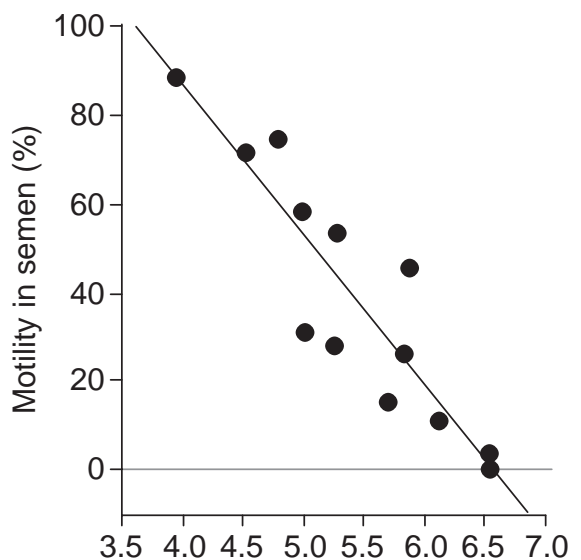

Log PMA-basal chemiluminescence (counts min $^{-1}$ ) (b) $y=-22.881 x+145.882, r=0.901$

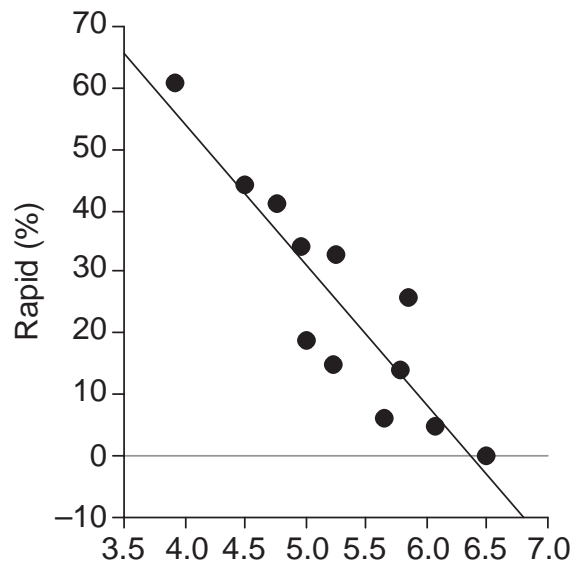

Log PMA-basal chemiluminescence (counts $\min ^{-1}$ )

(d) $y=-81.722 x+511.596, r=0.791$

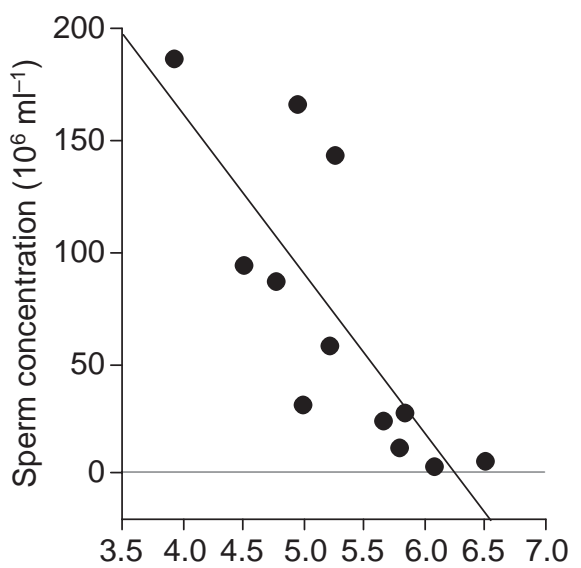

Log PMA-basal chemiluminescence (counts $\min ^{-1}$ )

Fig. 1. Relationships between the intensity of the signal generated by phorbol ester (PMA)positive leucocyte-free samples and semen quality. (a) Percentage of spermatozoa with progressive motility in semen. (b) Percentage of spermatozoa rapid in semen. (c) Percentage of spermatozoa motile in semen. (d) Concentration of spermatozoa in semen (Gomez et al., 1998).

et al., 1989). Moreover, human seminal plasma appears to contain sufficient free iron and copper to catalyse this process (Kwenang et al., 1987).

A second major feature of oxidative stress in the germ line is that its occurrence is profoundly influenced by the presence of antioxidants in the secretions of the male reproductive tract. These antioxidants include highly specialized protective enzymes that are secreted into the extracellular space. Examples include glutathione peroxidase (GPx5) and extracellular superoxide dismutase (SOD), produced in the caput and cauda epididymides, respectively (Perry et al., 1993; Vernet et al., 1996, 1997). Seminal plasma also contains small molecular mass free radical scavengers such as vitamin C, alpha tocopherol, tyrosine, hypotaurine and uric acid that contribute significantly to the antioxidant protection of spermatozoa (van Overveld et al. 2000). In addition, the albumin present in human seminal plasma is a sacrificial antioxidant that protects spermatozoa from peroxidative damage by absorbing lipid peroxides from the sperm plasma membrane (Twigg et al., 1998a). In view of the importance of oxidative stress in the aetiology of sperm dysfunction, it is not surprising that seminal antioxidant activity has been shown to be depressed in the ejaculates of infertile men and to exhibit an inverse correlation with fertility (Smith et al., 1996; Barbieri et al., 1999; Hendin et al., 1999). In addition, underexpression of mitochondrial phospholipid hydroperoxide glutathione peroxidase in spermatozoa has been linked to the motility 
loss exhibited in cases of asthenozoospermia (Imai et al., 2001).

The susceptibility of human spermatozoa to oxidative stress is exacerbated by the capacity of these cells to generate reactive oxygen species (ROS). When all traces of leucocyte contamination have been removed from the ejaculate, the amount of ROS generation correlates inversely with semen quality and sperm function (Aitken et al., 1992; Gomez et al., 1998). This association between ROS generation and poor semen quality is particularly evident when the spermatozoa are stimulated with phorbol esters (PMA). Normal, functional, mature human spermatozoa do not generate ROS in the presence of this reagent. However, in the infertile population, responses to PMA are obtained that correlate extremely well with the quality of the original ejaculate (Gomez et al., 1998; Fig 2). A powerful discriminator of infertility in patients not exhibiting leucocytospermia is a combined measure of ROS generation and antioxidant activity in the seminal plasma (Sharma et al., 1999). Analysis of the key antioxidant enzymes (SOD and catalase) in seminal plasma has not revealed any significant differences between the ejaculates of fertile and infertile men (Zini et al., 2000a). Thus, if there is an association between the overall antioxidant activity in seminal plasma and oxidative damage to the spermatozoa, it is presumably due to deficiencies in the presence of small molecular mass free radical scavengers such as vitamin C.

\section{Origins of oxidative stress}

The factors responsible for the excessive generation of ROS by the spermatozoa of infertile men have not yet been elucidated. In some cases, it may be the presence of xenobiotics that are induced to redox cycle by the spermatozoa and generate toxic free radicals. In other cases, there may be defects in the cellular mechanisms that normally regulate free radical generation by these cells. The most significant lead to date in resolving the aetiology of excess free radical generation by spermatozoa has been the observed association with defective cytoplasmic extrusion. Defective sperm function is associated with excessively high cellular concentrations of enzymes such as lactic acid dehydrogenase (Casano et al., 1991), creatine kinase (Huszar et al., 1988), SOD (Aitken et al., 1996) and glucose6-phosphate dehydrogenase (Aitken et al., 1994; Gomez et al., 1996). The feature that all these enzymes hold in common is that they are cytosolic. In keeping with this observation, the cellular content of these enzymes is correlated with the retention of excess residual cytoplasm by human spermatozoa (Gomez et al., 1996). The loss of sperm motility and fertilizing potential associated with varicocoeles and idiopathic male infertility is associated with the retention of excess residual cytoplasm by the spermatozoa (Zini et al., 1998, 1999, 2000b), as is the loss of fertility associated with heavy smoking (Mak et al., 2000). Recent studies of patients undergoing IVF therapy have demonstrated a strong negative correlation between fertilization rate and the presence of residual cytoplasm in the sperm midpiece (Keating et al., 1997).

In light of these observations, it is possible that the generation of excess free radicals by the spermatozoa of infertile patients reflects an underlying defect in Sertoli cell function, the latter failing to remove sufficient residual cytoplasm before spermatozoa are discharged from the germinal epithelium. The presence of excess residual cytoplasm then enhances the free radical generating system of the spermatozoa via mechanisms that are still poorly understood. One possibility is that the presence of excess glucose-6-phosphate dehydrogenase enhances the cellular generation of NADPH that, in turn, fuels the production of free radicals by a proposed sperm NADPH oxidase (Aitken et al., 1994). Further studies will be needed to determine whether this or other, equally plausible, mechanisms are involved.

\section{DNA damage}

Oxidative stress does not simply disrupt the fertilizing capacity of human spermatozoa, it also attacks the integrity of the DNA carried in the sperm nucleus and mitochondria. A variety of techniques has been used to demonstrate the presence of DNA fragmentation in human spermatozoa, including comet, nick translation and sperm chromatin structure assays (Hughes et al., 1996; Evenson et al., 1999; Irvine et al., 2000). DNA fragmentation appears to be inversely correlated with semen quality, particularly sperm count, morphology and motility (Shen et al., 1999; Irvine et al., 2000; Muratori et al., 2000; Shen and Ong, 2000). Moreover, negative correlations have been observed between the stability of DNA in the sperm nucleus and the fertilizing capacity of spermatozoa in vivo and in vitro (Sun et al., 1997; Aitken et al., 1998; Evenson et al., 1999; Host et al., 2000). The ability of the embryo to survive to term also appears to be negatively correlated with the level of DNA fragmentation in the germ line (Host et al., 2000).

That oxidative stress is correlated with DNA fragmentation has been demonstrated in many independent studies. Firstly, the DNA in the ejaculates of infertile men is commonly associated with oxidative damage as reflected by measurements of 8-hydroxydeoxyguanosine (8-OHdG) (Kodama et al., 1997; Irvine et al., 2000; Shen and Ong, 2000). Secondly, correlations have been observed between oxygen radical generation and DNA damage in ejaculated spermatozoa (Barroso et al., 2000; Irvine et al., 2000). Oxidative stress in the male germ line can also be promoted by the presence of transition metals such as iron, copper and nickel that stimulate free radical generation and DNA damage (Liang et al., 1999; Wellejus et al., 2000). Some protection against metal-catalysed DNA damage may be afforded by protamination of sperm chromatin. The $\mathrm{N}$ terminus of human protamine P2 contains a heavy metal trap with particular affinity for $\mathrm{Ni}(\mathrm{II})$ and $\mathrm{Cu}(\mathrm{II})$ (Liang et al., 1999). Therefore, protamines may serve a protective function by sequestering metals capable of promoting the 


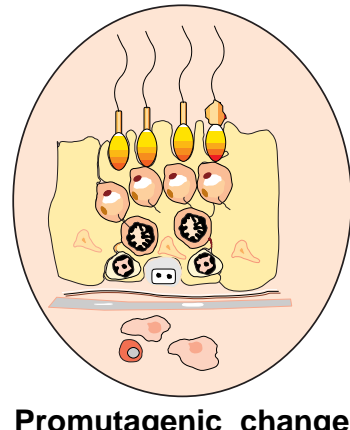

Promutagenic change

Spermatozoa emerging from the testes with oxidative base damage and

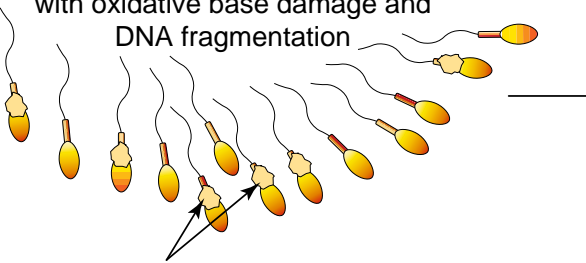

Presence of excess residual cytoplasm may be associated with excess

free radical generation

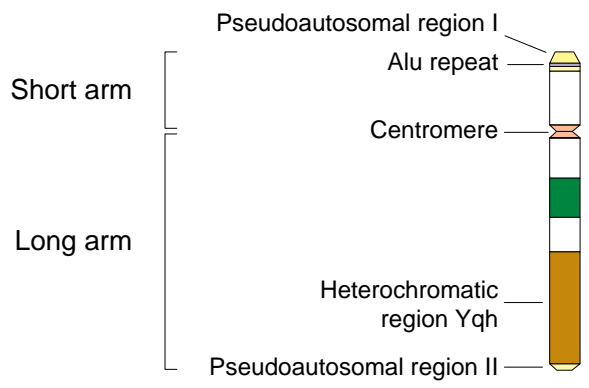

\section{DNA fragmentation induced in sperm} emerging from the testes as a result of:

Incomplete Fas-mediated apoptosis

Aberrant recombination

Aberrant chromatin repackaging

Redox cycling xenobiotics/heavy metals

Deficient antioxidant protection

Aberrant free radical generation

Aberrant spermiogenesis
Fig. 2. Proposed associations between DNA fragmentation in the male germ line and the aetiology of human disease. A variety of factors can conspire to induce oxidative stress and DNA damage in spermatozoa emerging from the male reproductive tract. It is proposed that this damage is promutagenic and can give rise to mutations after fertilization as the oocyte attempts to repair the DNA before initiation of the first cleavage division. Any mutations occurring at this point will be fixed in the germ line and may be responsible for the induction of pathology, such as infertility or childhood cancer, in the offspring. fragmentation of sperm DNA. This function may account in part for the extensive DNA damage observed in poorly packaged spermatozoa in which the protamine-histone transition has been incomplete (Bianchi et al., 1993).

An alternative aetiology for the DNA nicks seen in the spermatozoa of infertile patients involves an abortive apoptotic pathway mediated by Fas. The induction of apoptosis via the Fas pathway is clearly an important mechanism by which Sertoli cells regulate the number of germ cells, particularly in times of stress (Boekelheide et al., 2000). Accordingly, men exhibiting deficiencies in the semen profile, particularly oligozoospermia, possess a large number of spermatozoa bearing Fas, prompting the suggestion that these dysfunctional cells are the product of an incomplete apoptotic cascade (Sakkas et al., 1999b). Whether defective apoptosis accounts for a significant proportion of the DNA damage seen in the spermatozoa of infertile men is still an open question. A recent analysis of DNA damage in the germ line did not find ultrastructural evidence for apoptosis in association with DNA damage (Barroso et al., 2000), whereas another study found no correlation between DNA damage and Fas expression (Muratori et al., 2000). Of course, Fas binding and ROS generation are not mutually exclusive phenomena; ROS can induce Fas-mediated signal transduction in some types of cell (Huang et al., 2000), whereas Fas-induced apoptosis 
appears to be mediated by ROS in other types of cell (Sayers et al., 2000).

Double-stranded DNA breaks also occur naturally in the male germ line both in preparation for recombination and during the process of chromatin packaging (Sakkas et al., 1999a). These physiological strand breaks are normally resolved by the spermatid stage of spermatogenesis. Therefore, it is possible that aberrant recombinationchromatin packaging accounts for unresolved doublestrand breaks in mature human spermatozoa; however, evidence to support this contention is currently lacking.

\section{Consequences of DNA damage}

The studies cited above indicate that a variety of mechanisms, particularly oxidative stress, conspires to induce DNA strand breaks in the male germ line, particularly in cases of male subfertility associated with poor semen quality. Such samples frequently show depressed fertilization rates in vitro in association with the DNA damage (Sun et al., 1997), presumably as a consequence of collateral peroxidative damage to the sperm plasma membrane. Such membrane damage is physiologically important since it constitutes a protective mechanism designed to ensure that spermatozoa with severely damaged genomes cannot participate in the normal process of fertilization. However, this biological safeguard is of limited effectiveness because the genome of the human spermatozoon appears to be more susceptible than the plasma membrane to oxidative damage. As a consequence, it is possible to arrive at levels of oxidative stress at which cells exhibiting significant oxidative damage to their DNA retain the capacity for fertilization (Aitken et al., 1998).

\section{Clinical significance - childhood cancer}

The clinical significance of this situation is demonstrated by recent analyses of men who are heavy smokers. The ejaculates of such men are under oxidative stress as indicated by the fact that their semen is characterized by increased levels of oxidative DNA base damage, high chromatin fragmentation and low concentrations of antioxidant vitamins (Fraga et al., 1996). Although fertility is suppressed in such subjects, they are not infertile. As a consequence, DNA-damaged spermatozoa from heavy smokers are able to engage in the process of fertilization, with consequences for the ultimate health and well-being of the embryo. Thus, the offspring of heavy smokers are four to five times more likely to develop childhood cancer than the children of non-smoking fathers (Ji et al., 1997). Furthermore, the possible mutagenic-promutagenic effects of smoking on DNA integrity in the male germ line has been reinforced by another study indicating that $15 \%$ of all childhood cancers are directly attributable to paternal smoking (Sorahan et al., 1997). Evidence for this linkage between DNA damage in the male germ line and abnormalities in the developing embryo or child is not confined to smokers. It can also be found in the wealth of data indicating that powerful associations exist between childhood disease and paternal occupation (Sawyer and Aitken, 2000).

The use of ICSI as a therapeutic technique can only exacerbate this problem. The most highly damaged sperm DNA is generally found in men with the poorest semen quality, whose spermatozoa are incapable of fertilization (Irvine et al., 2000). This could be viewed as a protective mechanism ensuring that the most severely abnormal spermatozoa do not contribute their damaged DNA to the embryo. However, this safeguard is circumvented when ICSI is used to achieve human conceptions. Even if extremely high oxidative DNA damage is induced experimentally in populations of human spermatozoa by coincubation with activated leucocytes, exposure to hydrogen peroxide or the excessive stimulation of the free-radicalgenerating system of the spermatozoon itself with NADPH, successful fertilization can still be achieved with ICSI (Twigg et al., 1998b,c). Since DNA damage in the male germ line is associated with an increased incidence of childhood cancer, it is possible that the children of ICSI conceptions will be vulnerable to this disease. Much will depend on the DNA integrity of the spermatozoon selected for injection.

Childhood cancer may not be the only consequence of conceptions involving DNA-damaged spermatozoa. It is also possible that double-stranded DNA breakage induced by oxidative stress, defective apoptosis or aberrant recombination results in infertility in the male offspring as a consequence of irreparable deletions on the long arm of the Y chromosome.

\section{Y chromosome and male infertility}

The first association between spermatogenic failure and an underlying genetic cause was demonstrated by Tiepolo and Zuffardi (1976) in a report of six azoospermic patients carrying microscopically detectable deletions of the distal portion of Yq. In four cases, the deletion was de novo since the fathers of the patients were tested and found to carry an intact $Y$ chromosome. On the basis of this finding, the existence of a spermatogenesis factor, the 'azoospermia factor' (AZF) encoded by a gene on distal Yq, was proposed. However, it was not until the mid-1980s, when Y chromosome-specific probes were developed, that the regions associated with spermatogenic failure were defined. Vollrath et al. (1992) developed more than 200 sequence-tagged sites (STS: short tracts of DNA that act as a landmark to define position on a physical map) along the length of the $Y$ chromosome and ordered the STS markers using a large panel of individuals with $\mathrm{Y}$ chromosome deletions. These markers have permitted simple deletion analysis in infertile males by the polymerase chain reaction (PCR). Subsequently, many STS-based screening programmes have been undertaken in patients affected by azoospermia and severe oligozoospermia to define the AZF locus and isolate 
candidate genes for AZF. Vogt et al. (1996) observed that $Y$ chromosome microdeletions follow a certain deletion pattern, with three recurrently deleted nonoverlapping subregions in proximal, middle and distal $\mathrm{Yq} 11$, designated AZFa, AZFb and AZFC, respectively. In each AZF region, it has been suggested that the deletion is associated with a distinct histopathological profile. Thus, deletions removing the entire AZFa region result in type I Sertoli cell only syndrome (SCOS) (no germ cells present), deletions of the whole $\mathrm{AZFb}$ region result in spermatogenic arrest (SGA) usually at spermatocyte stage, and deletions in AZFC are associated with type II SCOS (some spermatogonia present with limited spermatogenesis) or hypospermatogenesis (oligozoospermia).

In each region, a number of candidate genes have been proposed (McElreavey and Krausz, 1999). No single genespecific deletion has been found in more than 200 oligospermic-azoospermic men screened for six AZF genes by PCR (Krausz et al., 1999a, 2001). This finding indicates that gene-specific deletions are probably rare events and only large $\mathrm{Y}$ deletions, removing several genes, are associated with the infertile phenotype. A large study on 576 infertile men describes a de novo point mutation of the gene USP9Y associated with non-obstructive azoospermia (Sun et al., 1999). This is the first formal demonstration for the aetiopathogenetic role of an AZF gene in spermatogenic failure.

Little is known about the biological functions of the proteins encoded by $\mathrm{Y}$ chromosome genes, although most seem to encode for proteins involved in RNA metabolism (DAZ, RBM, elF-1AY, DBY). During the later stages of spermiogenesis, when RNA synthesis is markedly reduced, post-transcriptional regulation plays a primary role. It is possible that factors encoded by $\mathrm{Y}$-linked genes have an important function in this process.

\section{Clinical significance of $\mathrm{Y}$ microdeletions}

The incidence of $\mathrm{Y}$ chromosome microdeletions among infertile men varies among studies from 1 to 55\% (Krausz and McElreavey, 1999). The major factor influencing this parameter is the composition of the study population. Since patients affected by idiopathic azoospermia are at higher risk for this genetic defect (approximately 15\% of azoospermic men have $\mathrm{Yq}$ microdeletions) than are patients affected by oligozoospermia (5-10\% oligozoospermic men have have $\mathrm{Yq}$ microdeletions), a higher proportion of azoospermic men in a given study population will be associated with a higher overall deletion frequency (Krausz et al., 1999b). Yq microdeletions are specific for spermatogenic failure since no microdeletions have been found in normospermic men (Krausz et al., 2001).

Y chromosome deletions are not completely incompatible with fertility because a few cases involving natural transmission of AZFc deletions from father to son have been described (Krausz and McElreavey, 2001; Saut et al., 2000). Since couple fertility is not a synonym of normozoospermia and sperm analysis of the fathers was not available, it is possible that these 'fertile fathers' may themselves have been oligozoospermic. However, it is also possible that environmental effects or different genetic backgrounds may account for a variable phenotype between a father and his son. For the same reason, the phenotype of an ICSI male offspring from a father with $\mathrm{Yq}$ deletions cannot be entirely predicted. $\mathrm{Yq}$ microdeletion has been found in two male children conceived through ICSI even though the deletion was not detected in the lymphocytes of their infertile father (Kent-First et al., 1996). Plausible explanations for this situation involve mosaicism in the father's germ line or high rates of DNA fragmentation in the spermatozoa followed by the creation of a post-fertilization Yq deletion. However, in a study of 99 ICSI-conceived sons, no de novo Yq deletion was found in the babies (Cram et al., 2000).

In contrast, patients carrying $\mathrm{Yq}$ deletions are associated with an increased incidence of $45, \mathrm{XO}$ cells in their lymphocytes and of sperm cells nullisomic for gonosomes, especially for the $Y$ chromosome (Siffroi et al., 2000). Therefore, $\mathrm{Yq}$ microdeletions may be associated with $\mathrm{Y}$ chromosomal instability leading to the formation of $45, \mathrm{XO}$ cell lines. These findings indicate a possible increased risk for Turner's syndrome in the offspring of men with $\mathrm{Yq}$ microdeletion.

A systematic Yq screening of ICSI male babies with longterm follow-up are warranted to understand more about the safety of this technique and the clinical consequence of the transmission of $\mathrm{Y}$ deletions. Since certain $\mathrm{Y}$ deletions (AZFC) are associated with a progressive change from oligozoospermia to azoospermia (Girardi et al., 1997; Simoni et al., 1997), preventive therapy (cryopreservation of spermatozoa for successive assisted reproductive techniques) could be proposed for affected sons.

\section{Origin and mechanism of $\mathrm{Y}$ chromosome microdeletions}

Apart from the few inherited cases cited above, most deletions occur as de novo events. The cellular origin of $Y$ chromosome deletions is not clear. Presumably, the deletions are preceded by double-strand breaks that, if they occurred premeiotically, could lead to the presence of $Y$ chromosome deletions in the ejaculated spermatozoa. Alternatively, the spermatozoa may harbour a high frequency of double-strand breaks that lead to the creation of deletions after fertilization.

The relatively high frequency of $Y$ deletions indicates that the $Y$ chromosome is particularly susceptible to the spontaneous loss of genetic material. The nature of this susceptibility remains speculative. One possibility is aberrant recombination events between areas of homologous or similar sequence repeats (for example, Alu repeats or gene families) between the $X$ and $Y$ chromosomes or within the $Y$ chromosome itself by unbalanced sister chromatid exchange (McElreavey and Krausz, 1999). The instability of the $\mathrm{Y}$ chromosome may also be related to the high 
frequency of repetitive elements clustered along the length of the $Y$ chromosome. Deletion interval 6 for example is rich in both inverted and direct repeats, many of which are several hundred kilobases in length (Yen, 1998). A subclass of AZFa deletions appears to have resulted from intrachromosomal crossovers between repeated human endogenous retroviral (HERV) sequences (Blanco et al., 2000; Kamp et al., 2000).

Finally, the particular vulnerability of the $Y$ chromosome to DNA deletions may be the result of its inability to participate in recombination repair. The DNA fragmentation that appears to be commonplace in spermatozoa (Aitken, 1999) has the potential to generate deletions as the chromatin unravels at fertilization. Any double-stranded DNA breaks would normally be repaired by homologous recombination in the few hours that elapse between fertilization and the initiation of the first cleavage division. However, this repair mechanism cannot apply to the nonrecombining region of the $Y$ chromosome, where the key spermatogenesis genes are housed and where recombination repair is impossible.

Notwithstanding the evident vulnerability of the $Y$ chromosome, the question remains as to why some men have AZF deletions and others do not. It has been proposed that a particular $Y$ chromosome sequence organization can facilitate deletion formation. However, analysis of $Y$ chromosome haplotypes in men with $Y$ deletions compared with controls with no deletion failed to identify any specific predisposing or protecting haplotype for or against $Y$ deletion formation (Quintana-Murci et al., 2001). In some pathologies characterized by single base-pair substitutions, a paternal age effect has been described. However, paternal age effects do not seem to contribute to the loss of $\mathrm{Y}$ sequences in most patients since the father's age at the time of conception of an infertile son with or without $\mathrm{Yq}$ deletions is not different from that of control men with normal Y chromosomes.

\section{Conclusions}

In conclusion, the male germ line appears to be particularly susceptible to mutagenic and promutagenic change. This variability is biological useful in that it creates the genetic diversity that fuels the evolutionary process, but the genetic instability characteristic of the male germ line is harmful in that it helps create the mutations responsible for genetic disease including cancer and infertility. The mutagenic mechanisms involve replication errors that are heavily correlated with paternal age and responsible for the spontaneous appearance of dominant genetic diseases such as Apert's syndrome and achondroplasia. In addition, the male germ line is highly susceptible to DNA fragmentation via mechanisms that are independent of paternal age. The aetiology of these strand breaks may involve aberrant recombination, defective chromatin packaging, abortive apoptosis and oxidative stress. Oxidative stress appears to be particularly important and may be induced by a variety of mechanisms including antioxidant depletion, redox cycling xenobiotics and defective cytoplasmic extrusion during spermiogenesis. Such oxidatively induced DNA fragmentation constitutes a promutagenic change that, in its most severe form, does not have an impact on the quality of the germ line because collateral oxidative damage to the sperm plasma membrane prevents fertilization. When there is less oxidative stress, fertilization can occur, but the oocyte must repair the DNA strand breaks before the initiation of the first cleavage division. It is at this juncture that deletions or sequence errors may be introduced (Fig. 2). The $\mathrm{Y}$ chromosome is particularly vulnerable to DNA damage, partly because of its genetic structure and partly because it cannot correct double-stranded DNA deletions by homologous recombination. The fact that such damage to the $\mathrm{Y}$ chromosome frequently results in infertility might be regarded as another safety mechanism that serves to limit the extent to which mutations are propagated in the germ line. If the DNA damage does not induce infertility through an effect on the $Y$ chromosome but involves an oncogene, the result will be an increased risk of cancer in the offspring. Such associations are illustrated by the increased risk of childhood cancer seen in the children of men who possess high DNA fragmentation in their spermatozoa as a consequence of heavy smoking. Moreover, because the mutation is fixed in the germ line, it has the potential to impact upon the health and well-being of all the future descendants of a given individual.

C. Krausz received a grant from the Italian Telethon (No. 281/6) and a travel grant from the University of Newcastle (Australia).

\section{References}

Key references are identified by asterisks

Agulnik AI, Bishop CE, Lerner JL, Agulnik SI and Solovyev VV (1997) Analysis of mutation rates in the SMCY/SMCX genes shows that mammalian evolution is male driven Mammalian Genome 8 134-138

*Aitken RJ (1999) The Amoroso Lecture: the human spermatozoon - a cell in crisis? Journal of Reproduction and Fertility 115 1-7

Aitken RJ, Clarkson JS and Fishel S (1989) Generation of reactive oxygen species, lipid peroxidation and human sperm function Biology of Reproduction 41 183-187

Aitken RJ, Buckingham D, West K, Wu FC, Zikopoulos K and Richardson DW (1992) Differential contribution of leucocytes and spermatozoa to the high levels of reactive oxygen species recorded in the ejaculates of oligozoospermic patients Journal of Reproduction and Fertility 94 451-462

Aitken RJ, Harkiss D and Buckingham DW (1993) Analysis of lipid peroxidation mechanisms in human spermatozoa Molecular Reproduction and Development 35 302-315

Aitken RJ, Krausz C and Buckingham DW (1994) Relationships between biochemical markers for residual sperm cytoplasm, reactive oxygen species generation and the presence of leucocytes and precursor germ cells in human sperm suspensions Molecular Reproduction and Development 39 268-279

Aitken RJ, Buckingham DW, Carreras A and Irvine DS (1996) Superoxide dismutase in human sperm suspensions: relationships with cellular composition, oxidative stress and sperm function Free Radical Biology and Medicine 21 495-504

Aitken RJ, Gordon E, Harkiss D, Twigg JP, Milne P, Jennings Z and Irvine DS (1998) Relative impact of oxidative stress on the functional 
competence and genomic integrity of human spermatozoa Biology of Reproduction 59 1037-1046

Barbieri ER, Hidalgo ME, Venegas A, Smith R and Lissi EA (1999) Varicocele-associated decrease in antioxidant defenses Journal of Andrology 20 713-717

Barroso G, Morshedi M and Oehninger S (2000) Analysis of DNA fragmentation, plasma membrane translocation of phosphatidylserine and oxidative stress in human spermatozoa Human Reproduction 15 1338-1344

Bianchi P, Manicardi GC, Bizzaro D, Bianchi U and Sakkas D (1993) Effect of DNA protamination on fluorochrome staining and in situ nicktranslation of murine and human mature spermatozoa Biology of Reproduction 49 1038-1043

Birky CW, Jr (1995) Uniparental inheritance of mitochondrial and chloroplast genes: mechanisms and evolution Proceedings of the National Academy of Sciences USA $9211331-11338$

Blanco P, Shlumukova M, Sargent CA, Jobling MA, Affara N and Hurles ME (2000) Divergent outcomes of intrachromosomal recombination on the human $\mathrm{Y}$ chromosome: male infertility and recurrent polymorphism Journal of Medical Genetics 37 752-758

Boekelheide K, Fleming SL, Johnson KJ, Patel SR and Schoenfeld HA (2000) Role of Sertoli cells in injury-associated testicular germ cell apoptosis Proceedings of the Society for Experimental Biology and Medicine 225 $105-115$

Burgoyne PS (1998) The mammalian Y chromosome: a new perspective Bioessays 20 363-366

Casano R, Orlando C, Serio M and Forti G (1991) LDH and LDH-X activity in sperm from normospermic and oligozoospermic men International Journal of Andrology 14 257-263

Chai NN, Salido EC and Yen PH (1997) Multiple functional copies of RBM gene family, a spermatogenesis candidate on the human $\mathrm{Y}$ chromosome Genomics 45 355-361

Cram DS, Ma K, Bhasin S, Ariasc J, Pandjaitanc M, Chu B, Audrins MS, Saunders D, Quinn F, deKretser D and McLachlan R (2000) Y chromosome analysis of infertile men and their sons conceived through intracytoplasmic sperm injection: vertical transmission of deletions and rarity of de novo deletions Fertility and Sterility 74 909-915

*Crow JF (1997) The high spontaneous mutation rate: is it a health risk? Proceedings of the National Academy of Sciences USA 94 8380-8386

Cummins JM (2000) Fertilization and elimination of the paternal mitochondrial genome Human Reproduction 15 Supplement 2 92-101

Delbridge ML and Graves JA (1999) Mammalian Y chromosome evolution and the male-specific functions of $\mathrm{Y}$ chromosome-borne genes Reviews of Reproduction 4 101-109

Donnelly ET, O'Connell M, McClure N and Lewis SE (2000) Differences in nuclear DNA fragmentation and mitochondrial integrity of semen and prepared human spermatozoa Human Reproduction 15 1552-1561

Evenson DP, Jost LK, Marshall D, Zinaman MJ, Clegg E, Purvis K, de Angelis P and Claussen OP (1999) Utility of the sperm chromatin structure assay as a diagnostic and prognostic tool in the human fertility clinic Human Reproduction 14 1039-1049

Fraga CG, Motchnik PA, Wyrobek AJ, Rempel DM and Ames BN (1996) Smoking and low antioxidant levels increase oxidative damage to DNA Mutation Research 351 199-203

Girardi SK, Mielnik A and Schlegel PN (1997) Submicroscopic deletions in the $Y$ chromosome of infertile men Human Reproduction 12 1635-1641

Gomez E, Buckingham DW, Brindle J, Lanzafame F, Irvine DS and Aitken RJ (1996) Development of an image analysis system to monitor the retention of residual cytoplasm by human spermatozoa: correlation with biochemical markers of the cytoplasmic space, oxidative stress and sperm function Journal of Andrology 17 276-287

Gomez E, Irvine DS and Aitken RJ (1998) Evaluation of a spectrophotometric assay for the measurement of malondialdehyde and 4hydroxyalkenals in human spermatozoa: relationships with semen quality and sperm function International Journal of Andrology 21 81-94

Hales DB, Diemer T and Hales KH (1999) Role of cytokines in testicular function Endocrine 10 201-217

Hendin BN, Kolettis PN, Sharma RK, Thomas AJ, Jr and Agarwal A (1999) Varicocele is associated with elevated spermatozoal reactive oxygen species production and diminished seminal plasma antioxidant capacity Journal of Urology 161 1831-1834

Host E, Lindenberg S and Smidt-Jensen S (2000) The role of DNA strand breaks in human spermatozoa used for IVF and ICSI Acta Obstetrica Gynecologica Scandinavia 79 559-563

Huang C, Li J, Zheng R and Cui K (2000) Hydrogen peroxide-induced apoptosis in human hepatoma cells is mediated by CD95(APO-1/Fas) receptor/ligand system and may involve activation of wild-type p53 Molecular Biology Reports 27 1-11

Hughes CM, Lewis SEM, McKelvey-Martin J and Thompson W (1996) A comparison of baseline and induced DNA damage in human spermatozoa from fertile and infertile men, using a modified comet assay Molecular Human Reproduction 2 613-619

Huszar G, Vigue L and Corrales M (1988) Sperm creatine phosphokinase quality in normospermic, variablespermic and oligospermic men Biology of Reproduction 38 1061-1066

Imai H, Suzuki K, Ishizaka K, Ichinose S, Oshima H, Okayasu I, Emoto K, Umeda M and Nakagawa Y (2001) Failure of the expression of phospholipid hydroperoxide glutathione peroxidase in the spermatozoa of human infertile males Biology of Reproduction 64 674-683

Irvine DS, Twigg J, Gordon E, Fulton N, Milne P and Aitken RJ (2000) DNA integrity in human spermatozoa: relationship with semen quality Journal of Andrology 21 33-44

*Ji BT, Shu XO, Linet MS, Zheng W, Wacholder S, Gao YT, Ying DM and Jin F (1997) Paternal cigarette smoking and the risk of childhood cancer among offspring of nonsmoking mothers Journal of the National Cancer Institute 89 238-244

Jones R, Mann T and Sherins RJ (1979) Peroxidative breakdown of phospholipids in human spermatozoa: spermicidal effects of fatty acid peroxides and protective action of seminal plasma Fertility and Sterility 31 531-537

Kamp C, Hirschmann P, Voss H, Huellen K and Vogt PH (2000) Two long homologous retroviral sequence blocks in proximal Yq11 cause AZFa microdeletions as a result of intrachromosomal recombination events Human Molecular Genetics 9 2563-2572

Kao SH, Chao HT and Wei YH (1998) Multiple deletions of mitochondrial DNA are associated with the decline of motility and fertility of human spermatozoa Molecular Human Reproduction 4 657-666

Keating J, Grundy CE, Fivey PS, Elliott M and Robinson J (1997) Investigation of the association between the presence of cytoplasmic residues on the human sperm midpiece and defective sperm function Journal of Reproduction and Fertility 110 71-77

Kent-First MG, Kol S, Muallem A, Ofir R, Manor D, Blazer S, First N and Itskovitz-Eldor J (1996) the incidence and possible relevance of Y-linked microdeletions in babies born after intracytoplasmic sperm injection and their infertile fathers Molecular Human Reproduction 2 943-945

Kodama H, Yamaguchi R, Fukuda J, Kasai H and Tanaka T (1997) Increased oxidative deoxyribonucleic acid damage in the spermatozoa of infertile male patients Fertility and Sterility 68 519-524

Krausz C and McElreavey K (1999) Y chromosome and male infertility Frontiers in Bioscience 4 1-8

Krausz C and McElreavey K (2001) Y chromosome microdeletions in "fertile" males Human Reproduction 161306

Krausz C, Bussani-Mastellone C, Granchi S, McElreavey K, Scarselli G and Forti G (1999a) Screening for microdeletions of Y chromosome genes in patients undergoing intracytoplasmic sperm injection Human Reproduction 14 1717-1721

*Krausz C, Quintana-Murci L, Barbaux S et al. (1999b) A high frequency of $\mathrm{Y}$ chromosome deletions in males with nonidiopathic infertility Journal of Clinical Endocrinology and Metabolism 84 3606-3612

Krausz C, Rajpert-De Meyts E, Frydelund-Larsen L, Quintana-Murci L, McElreavey K and Skakkebaek NE (2001) Double blind Y chromosome microdeletion analysis in men with known sperm parameters and reproductive hormone profiles: microdeletions are specific for spermatogenic failure Journal of Clinical Endocrinology and Metabolism 86 2638-2642

Kwenang A, Kroos MJ, Koster JF and Van Eijk HG (1987) Iron, ferritin and copper in seminal plasma Human Reproduction 2 387-388

Lahn BT and Page DC (1997) Functional coherence of the human Y chromosome Science $\mathbf{2 7 8}$ 675-680 
Lahn BT and Page DC (1999) Retroposition of autosomal mRNA yielded testis-specific gene family on human Y chromosome Nature Genetics 21 429-433

Liang R, Senturker S, Shi X, Bal W, Dizdaroglu M and Kasprzak KS (1999) Effects of $\mathrm{Ni}(\mathrm{II})$ and $\mathrm{Cu}(\mathrm{II})$ on DNA interaction with the $\mathrm{N}$-terminal sequence of human protamine $\mathrm{P} 2$ : enhancement of binding and mediation of oxidative DNA strand scission and base damage Carcinogenesis 20 893-898

McElreavey K and Krausz C (1999) Male infertility and the Y chromosome American Journal of Human Genetics 64 928-933

MacLeod J (1943) The role of oxygen in the metabolism and motility of human spermatozoa American Journal of Physiology 138 512-518

Mak V, Jarvi K, Buckspan M, Freeman M, Hechter S and Zini A (2000) Smoking is associated with the retention of cytoplasm by human spermatozoa Urology 56 463-466

Marshall Graves J (2000) Human Y chromosome, sex determination, and spermatogenesis - a feminist view Biology of Reproduction 63 667-676

Muratori M, Piomboni P, Baldi E, Filimberti E, Pecchioli P, Moretti E, Gambera L, Baccetti B, Biagiotti R, Forti G and Maggi M (2000) Functional and ultrastructural features of DNA-fragmented human sperm Journal of Andrology 21 903-912

Perry AC, Jones R and Hall L (1993) Isolation and characterization of a rat cDNA clone encoding a secreted superoxide dismutase reveals the epididymis to be a major site of its expression Biochemical Journal 293 21-25

Quintana-Murci L, Krausz C, Heyer E et al. (2001) The relationship between $Y$ chromosome DNA haplotypes and $Y$ chromosome deletions leading to male infertility Human Genetics 108 55-58

Reynier P, Chretien MF, Savagner F, Larcher G, Rohmer V, Barriere P and Malthiery $\mathbf{Y}$ (1998) Long PCR analysis of human gamete mtDNA suggests defective mitochondrial maintenance in spermatozoa and supports the bottleneck theory for oocytes Biochemical and Biophysical Research Communications 252 373-377

Sakkas D, Mariethoz E, Manicardi G, Bizzaro D, Bianchi PG and Bianchi U (1999a) Origin of DNA damage in ejaculated human spermatozoa Reviews of Reproduction 4 31-37

Sakkas D, Mariethoz E and St John JC (1999b) Abnormal sperm parameters in humans are indicative of an abortive apoptotic mechanism linked to the Fas-mediated pathway Experimental Cell Research 251 350-355

Saut N, Terriou P, Navarro A, Levy N and Mitchell MJ (2000) The human Y chromosome genes BPY2, CDY1 and DAZ are not essential for sustained fertility Molecular Human Reproduction 6 789-793

Sawyer DE and Aitken RJ (2000) Male mediated developmental defects and childhood disease Reproductive Medicine Review 8 107-126

Saxena R, Brown LG, Hawkins T, Alagappan RK, Skaletsky H, Reeve MP, Reijo R, Rozen S, Dinulos MB, Disteche CM and Page DC (1996) The DAZ gene cluster on the human $Y$ chromosome arose from an autosomal gene that was transposed, repeatedly amplified and pruned Nature Genetics 14 292-299

Sayers TJ, Brooks AD, Seki N, Smyth MJ, Yagita H, Blazar BR and Malyguine AM (2000) T cell lysis of murine renal cancer: multiple signaling pathways for cell death via Fas Journal of Leukocyte Biology $6881-86$

Sharma RK, Pasqualotto FF, Nelson DR, Thomas AJ and Agarwal A (1999) The reactive oxygen species-total antioxidant capacity score is a new measure of oxidative stress to predict male infertility Human Reproduction 14 2801-2807

Shen H and Ong C (2000) Detection of oxidative DNA damage in human sperm and its association with sperm function and male infertility Free Radicals in Biology and Medicine 28 529-536

Shen HM, Chia SE and Ong CN (1999) Evaluation of oxidative DNA damage in human sperm and its association with male infertility Journal of Andrology 20 718-723

Shimmin LC, Chang BH and Li WH (1993) Male-driven evolution of DNA sequences Nature $362745-747$

Short RV (1997) The testis - the witness of the mating system, the site of mutation and the engine of desire Acta Paediatrica 86 3-7

Siffroi JP, Le Bourhis C, Krausz C et al. (2000) Sex chromosome mosaicism in males carrying $\mathrm{Y}$ chromosome long arm deletions Human Reproduction 15 2559-2562
Simoni M, Gromoll J, Dworniczak B et al. (1997) Screening for deletions of the $\mathrm{Y}$ chromosome involving the $D A Z$ (deleted in azoospermia) gene in azoospermia and severe oligozoospermia Fertility and Sterility $\mathbf{6 7}$ 542-547

Smith R, Vantman D, Ponce J, Escobar J and Lissi E (1996) Total antioxidant capacity of human seminal plasma Human Reproduction 11 1655-1660

Sorahan T, Lancashire RJ, Hulten MA, Peck I and Stewart AM (1997) Childhood cancer and parental use of tobacco: deaths from 1953 to 1955 British Journal of Cancer 75 134-138

Sun C, Skaletsky H, Birren B, Devon K, Tang Z, Silber S, Oates R and Page DC (1999) An azoospermic man with a de novo point mutation in the $Y$ chromosomal gene USP9Y Nature Genetics 23 429-432

Sun JG, Jurisicova A and Casper RF (1997) Detection of deoxyribonucleic acid fragmentation in human sperm: correlation with fertilization in vitro. Biology of Reproduction 56 602-607

Tiepolo L and Zuffardi $\mathbf{O}$ (1976) Localization of factors controlling spermatogenesis in the nonfluorescent portion of the human $Y$ chromosome long arm Human Genetics 34 119-124

Twigg J, Irvine DS, Houston P, Fulton N, Michael L and Aitken RJ (1998a) latrogenic DNA damage induced in human spermatozoa during sperm preparation: protective significance of seminal plasma Molecular Human Reproduction 4 439-445

*Twigg J, Fulton N, Gomez E, Irvine DS and Aitken RJ (1998b) Analysis of the impact of intracellular reactive oxygen species generation on the structural and functional integrity of human spermatozoa: lipid peroxidation, DNA fragmentation and effectiveness of antioxidants Human Reproduction 13 1429-1437

Twigg JP, Irvine DS and Aitken RJ (1998c) Oxidative damage to DNA in human spermatozoa does not preclude pronucleus formation at ICSI Human Reproduction 13 1864-1871

van Overveld FW, Haenen GR, Rhemrev J, Vermeiden JP and Bast A (2000) Tyrosine as important contributor to the antioxidant capacity of seminal plasma Chemical and Biological Interactions 127 151-161

Vernet P, Rigaudiere N, Ghyselinck N, Dufaure JP and Drevet JR (1996) In vitro expression of a mouse tissue-specific glutathione peroxidase protein lacking selenocysteine can protect stably transfected mammalian cells against oxidative damage Biochemistry and Cell Biology $\mathbf{7 4}$ 125-131

Vernet P, Faure J, Dufaure JP and Drevet JR (1997) Tissue and developmental distribution, dependence upon testicular factors and attachment to spermatozoa of GPX5, a murine epididymis-specific glutathione peroxidase Molecular Reproduction and Development 47 87-98

Vogt PH, Edelmann A, Kirsch S et al. (1996) Human $\mathrm{Y}$ chromosome azoospermia factors (AZF) mapped to different subregions in Yq11 Human Molecular Genetics 5 933-943

Vollrath D, Foote S, Hilton A, Brown LG, Beer-Romero P, Bogan IS and Page DC (1992) The human Y chromosome: a 43-interval map based on naturally occurring deletions Science 258 52-59

Waters PD, Duffy B, Frost CJ, Delbridge ML and Graves JA (2001) The human $\mathrm{Y}$ chromosome derives largely from a single autosomal region added to the sex chromosomes 80-130 million years ago Cytogenetics and Cell Genetics 92 74-79

Wellejus A, Poulsen HE and Loft S (2000) Iron-induced oxidative DNA damage in rat sperm cells in vivo and in vitro. Free Radical Research 32 $75-83$

Yen PH (1998) A long range restriction map of deletion interval 6 of the human $\mathrm{Y}$ chromosome: a region frequently deleted in azoospermic males Genomics 54 5-12

Zini A, O'Bryan MK, Israel L and Schlegel PN (1998) Human sperm NADH and NADPH diaphorase cytochemistry: correlation with sperm motility Urology 51 464-468

Zini A, Buckspan M, Jama IM and Jarvi K (1999) Effect of varicocelectomy on the abnormal retention of residual cytoplasm by human spermatozoa Human Reproduction 14 1791-1793

Zini A, Garrels K and Phang D (2000a) Antioxidant activity in the semen of fertile and infertile men Urology 55 922-926

Zini A, Defreitas G, Freeman M, Hechter S and Jarvi K (2000b) Varicocele is associated with abnormal retention of cytoplasmic droplets by human spermatozoa Fertility and Sterility 74 461-464 